\title{
Arbeitsgruppe Externe Fixation der Deutschen Sektion der Internationalen Arbeitsgemeinschaft für Osteosynthesefragen
}

Leitung: Prof. Dr. med. D. Höntzsch

Mitglieder: Dr. med. G. Böhmer, Duisburg - Prof. Dr. med. L. Claes, Ulm · Prof. Dr. med. A. Dávid, Wuppertal · Prof. Dr. med. CH. Eggers, Hamburg · Dr. med. R. Friedel, Jena · Prof. Dr. med. H. Gerngroß, Ulm · Dr. med. R. Grass, Dresden · Dr. med. F. Kisse, Neustadt · Dr. med. H. Schmickal, Ludwigshafen . Dr. med. J. Schmidt, Erfurt · Dr. med. G. Suger, Ulm · Prof. Dr. med. A. Wentzensen, Ludwigshafen

\section{Zusammenfassung}

Die Arbeitsgruppe Externe Fixation arbeitet an Grundlagenforschung, Studien, Koordinationsvorschlägen und vor allem an der Entwicklung von Instrumenten und Implantaten. Die Arbeitsgruppe schätzt sich glücklich, dass einige ihre Projekte weltweit marktreif geworden sind und erhältlich sind und dass die Arbeitsgruppe an vielen Projekten des AO-Entwicklungsinstitutes und der Synthesfirmen der Gestaltung mithelfen konnte, dass hier ebenfalls Projekte marktreif geworden sind.
Beispiel für erfolgreich abgeschlossene Projekte sind: Neue Kombinationsbakke mit Snap-on Funktion, monolaterales externes Fixationssystem für Orthopädie, und Traumatologie (MEFISTO), neue offene Snap- on Universalbacke und Fractometerstudie.

Als Zukunftsprojekte sind zu nennen: Externe Repositionssysteme, Bewegungsbacken, röntgendurchlässige Fixateurbacken, intelligente Fixteurkomponenten, Kuhlbüchse, beschichtete Schanzsche Schrauben und als ein besonderes Anliegen Koordination und „Familienbildung“ aller Fixateur externe - Systeme der AO.

\section{Einleitung}

Die Arbeitsgruppe Externe Fixation der Deutschen Sektion der Internationalen Arbeitsgemeinschaft für Osteosynthesefragen ist ein seit Jahren im Sinne und im Auftrag der Arbeitsgemeinschaft für Osteosynthesefragen arbeitende Arbeitsgruppe und Gremium. In seiner Zusammensetzung hat diese eine gute Kontinuität der Mitglieder und wenig Fluktuation. In den ersten Jahren war Prof. Dr. Wentzensen aus Ludwigshafen der Leiter, seit 1996 Prof. Dr. Höntzsch, Tübingen.

Seit 1998 ist die Arbeitsgruppe nicht zuletzt dank ihrer tatsächlichen praxisnahen Erfolge gleichzeitig working-group (Arbeitsgruppe) der AOTK. Hierdurch können in beide Richtungen, d.h. von der Arbeitsgruppe zur AO sowie von der AO zur Arbeitsgruppe, Synergieeffekte er-

OP-JOURNAL 2000; 16: 276-281

(C) Georg Thieme Verlag Stuttgart · New York

zielt werden. Und es werden die Untersuchungen und Ergebnisse gebündelt und Doppeluntersuchungen und -entwicklungen vermieden. Die Arbeitsgruppe tagt im Regelfall 2-mal im Jahr.

\section{Projekte}

Kombinationsbacke/combination clamp/CC (Abb.1)

Die Akzeptanz einerseits sowie das tagtägliche (nachtnächtliche) Handling eines Fixateur externe hängt vor allem mit der Handhabbarkeit der Verbindungsbacken zwischen Rohren und Schanzscher Schraube sowie Rohr-zu-Rohr bei der Modulartechnik ab. Deshalb wird der Weiterentwicklung von vorhandenen Backen und Neuentwicklung von Backen große Bedeutung zugemessen. Zusammen mit Synthes USA wurde eine Backe, deren Grundkonzept dort bei Synthes USA/Paoli angestoßen wurde, entwickelt. Das Grundkonzept sieht vor, dass

a) für alle Verbindungen, d.h. zwischen Rohr und Rohr sowie zwischen Rohr und Pin, nur noch eine Backe verwenden werden muss und somit das Sorti- ment nur noch aus einem Backentyp bestehen würde,

b) alle Verbindungen seitlich offen sind,

c) eine Federspannung ermöglicht, dass die Backen aufgeschnappt werden können sowie dann in dieser Position zwar mit leichter Kraft verschieblich, aber an der einmal eingestellten Position haften bleiben (Snap-on-Effekt).

In den Jahren 1997 und 1998 wurde zunächst über mehrfach durchgeführte Handling-Tests das primäre Design so weit verbessert, dass an klinische Tests und an klinische Anwendung gedacht werden konnte.

Diese wurden mit drei sich ändernden und in jedem Fall verbessernden Prototypenserien vor allem in der BG-Unfallklinik Tübingen bei diversen Fixateuren angewendet. Es wurden hierbei Fixateure mit Elektivanwendung sowie Unfallanwendung verwendet.

Anfänglich wurden nur einzelne Backen für eine Fixateurmontage benutzt, um etwaige Defizite durch diese Backe durch die anderen Backen im System zu kompensieren. Es zeigte sich aber sofort, dass dies nicht notwendig ist, so dass in der zweiten und dritten Phase auch Fixateur-Externe-Montagen ausschließlich mit den neuen Kombinationsbacken durchgeführt wurden.

Es zeigte sich von Anfang an und mit den eingebauten Verbesserungen immer mehr, dass diese Backe enorme Handlingvorteile mit sich bringt und ein deutlicher Fortschritt ist. Anfänglich hat vor allem der Autor gedacht, dass die Kombinationsbacke für die ja häufigere Anwendung der Verbindung zwischen Rohr und Pin zu klobig wäre bzw. zu klobig aussehen könnte. Im Gesamtaspekt eines Fixateur externe mit diesen neuen Kombinationsbacken hat sich dies dann aber nicht bewahrheitet, so dass von dieser Seite her kein Argument gegen die Anwendung gesprochen hat. Die Feinarbeit 

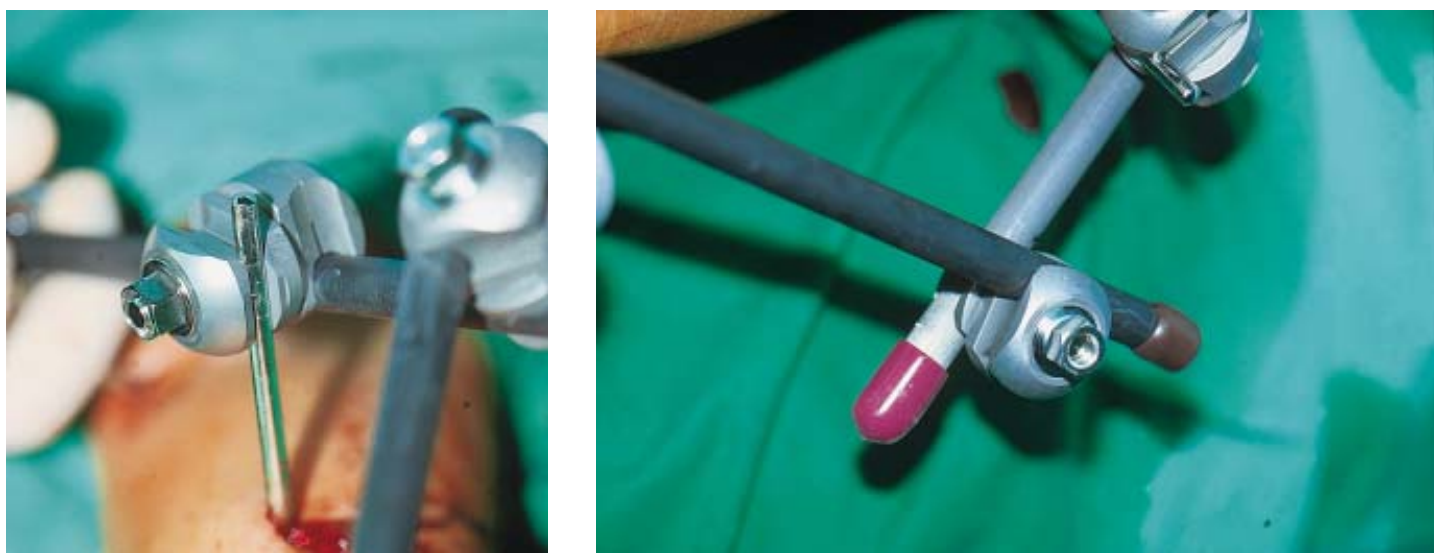

Abb.1 Kombinationsbacke/Combination Clamp/CC. Diese Backe hat für alle Funktionen einen „Snap-on“-Effekt. Mit dieser Backe können Rohr-Rohr bzw. Kohlefaserstab-Kohlefaserstab sowie Rohr- bzw. Stab-Schanzscher Schraube $4-6$ mm verbunden werden. Die Vorteile sind: Snap-on Effekt, gute Rohr-Rohr-Verbindung, tolerant gegen verschiedene Toleranzen mit alten und neuen Stäben/Rohren und man braucht nur eine Backe auf dem Sieb, da alle Verbindungen möglich sind.

an der Backe mit der leichten und symmetrischen Einschiebbarkeit von Rohren bzw. Rohr/Pin-Kombinationen hat dann letztendlich noch enorme Kraft, Engagement und Zeit gekostet. Hierzu waren neben den Handlingtests im Operationssaal dann vor allem auch die Handlingtests und Beratung aller Mitglieder der Arbeitsgruppe sehr hilfreich.

Mitte 1998 konnte dann das Genehmigungsverfahren in der Longbone-TK und in der AOTK als letztes Einscheidungsgremium zügig durchgezogen werden, so dass die Backe als offizielles Synthesprodukt seit Herbst 1998 zur Verfügung steht.

Die Kombinationsbacke ist in den USA ein beachtenswerter Verkaufserfolg. Sie ist für das Jahr 2000 in den europäischen Synthes-Katalog aufgenommen worden. Mit diesem Erfolg ist das Projekt Kombinationsbacke erfolgreich abgeschlossen.

Es steht eine neue Kombinationsbacke für den Fixateur externe zur Verfügung:

a Alle Funktionen:

$11 \mathrm{~mm}$ Rohr/Stab

mit $11 \mathrm{~mm}$ Rohr/Stab oder

$11 \mathrm{~mm}$ Rohr/Stab mit 4-6 mm Pin.

- Aufschnappbar, selbsthaltend („Snap-on“)

- Seitlich offen. Tolerant für Durchmessertoleranzen.

- Auf Pin und Rohr/Stab selbsthaltend.

\section{Fraktometerstudie}

Die Fraktometerstudie wird seit 4 Jahren durchgeführt. Es machen alle Kliniken außer Tübingen mit. Das Studienziel ist dadurch gekennzeichnet:
Ist es möglich, mit einem äußeren Messgerät, das von Zeit zu Zeit Mikrobewegungen unter definierter Belastung misst, folgende Fragen zu beantworten:

1. Gibt es einen frühestmöglichen Zeitpunkt, um zu beurteilen, ob eine Ausbehandlung im Fixateur externe erfolgreich ist oder ob es zu einer der gefürchteten Nichtheilungen kommt?

2. Ist es möglich, mit diesem Messgerät Röntgenuntersuchungen bei der Ausheilung, d.h. letztendlich dem Zeitpunkt der Abnahme des Fixateur externe, einzusparen?

3. Kann aufgrund der nichtheilenden Fälle herausgefunden werden, welche Kriterien womöglich für eine Nichtheilung im Fixateur externe maßgeblich anzuschuldigen sind, und wie diese gegebenenfalls abzustellen sind.

\section{Methode}

Von Prof. Claes als einem Mitglied der Gruppe wurde ein Bewegungsmessgerät entwickelt, welches auf kleinste Bewegungen anspricht und zwischen den proximalen und distalen Schanzschen Schrauben am Fixateur eingesetzt werden kann.

Zum ausgesuchten Messzeitpunkt wird dann eine definierte Last, entweder axiale Belastung $20 \mathrm{~kg}$ oder Anheben des Beines, vorgenommen und der Messwert bestimmt. Der erste Messwert bei noch nicht eingetretener Heilung wird als $100 \%$ angenommen. Die weitere Abnahme wird dann in abnehmenden Prozentzahlen angegeben.

\section{Material}

Aus den Kliniken Hamburg, Ludwigshafen und Ulm wurden prospektiv alle Patienten mit einer Fixateur externe-Behandlung ausgesucht, um in die Studie aufgenommen zu werden. Nähere Einzelheiten gibt dann die ausführliche Studie, welche in einem vorläufigen Bericht aus dem Institut und in der zu erwartenden Publikation dargestellt wird. Letztendlich war 1998 dann die Fallzahl $n=103$ eingetreten.

\section{Ergebnisse}

Es zeigt sich, dass es in $8 \%$ zum Heilungsversagen kommt. Die Kurven trennen sich eindeutig in die Gruppe der Heiler und der Nichtheiler. Die Trennung ist zwischen 4 und 6 Wochen erkennbar.

Die heilenden Frakturen enden in einem asymptomatischen Plateau, wo die Bewegung nicht mehr weiter zunimmt, so dass dieser Zeitpunkt spätestens nach drei, aber häufig bereits nach zwei Messungen, die auf gleichem Niveau liegen, ohne weitere Röntgenkontrolle bestimmt werden kann.

Die dritte Frage der Studie nach Gründen der Nichtheilung konnte an den acht Fällen der Nichtheilung zumindest in einem Bereich klar erkannt werden:

Einfache Frakturformen sollten möglichst gut reponiert werden, da mit steigendem Frakturspalt die Heilungszeit zunimmt bzw. eine Nichtheilung zu erwarten ist. Für komplexe Frakturen lässt sich keine Korrelation erarbeiten. 


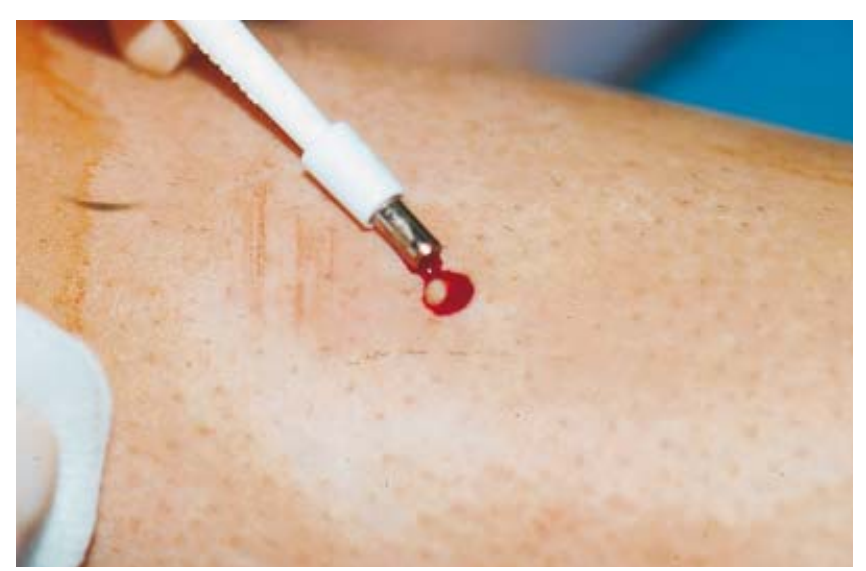

Abb. 2 Hautstanze. Bei Eintrittstellen für Schanzsche Schrauben, bei denen nicht viel Relativbewegung zu erwarten ist (z. B. am Unterschenkel) bewähren sich runde Hautstanzen. Wir verwenden $1 \mathrm{~mm}$ dünnere als die jeweilige Schanzsche Schraube. D. h. bei einer $5 \mathrm{~mm}$ Schanzschen Schraube eine 4-mm-Stanze.

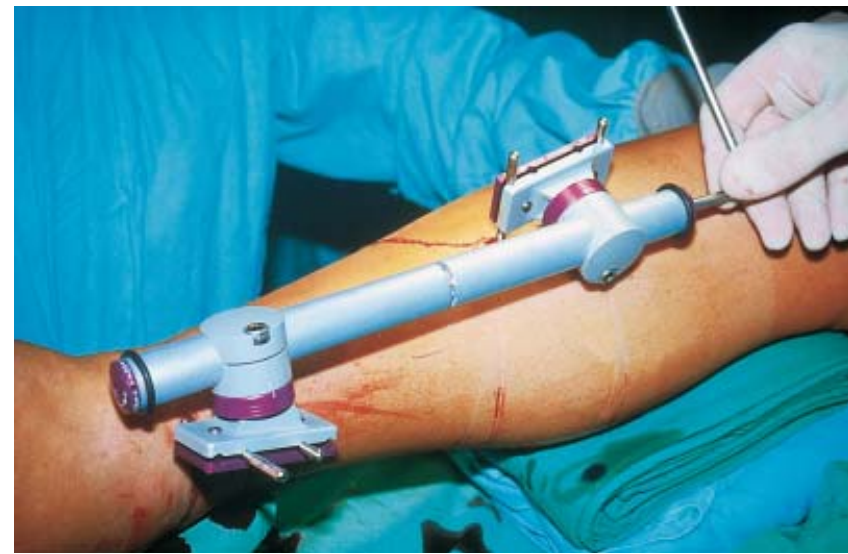

Abb.3 MEFISTO (Monolaterales Externes Fixationssystem für Orthopädie und Traumatologie). Ein Beispiel am Unterschenkel.

\section{Schlussfolgerung}

1. Das Fractometer-Messgerät ist geeignet, zwischen der 4. und 6. Woche nichtheilende Frakturen im Fixateur externe zu erkennen und zu diesem Zeitpunkt einen Verfahrenswechsel zu empfehlen.

2. Das Festwerden der Fraktur lässt sich mit dem Fractometer erkennen, so dass zu diesem Zeitpunkt Röntgenkontrollen eingespart werden können, d.h. weniger Röntgenkontrollen, weiterhin die klinische Kontrolle, das subjektive Empfinden und letztendlich die kontinuierliche Fractometermessung können helfen, den richtigen Zeitpunkt der Fixateurexterne-Abnahme ohne weitere Röntgenkontrolle herauszufinden.

Am Fixateur lässt sich mit geeigneten Methoden die Frakturheilung messen.

- Als geeignetes Instrument hat sich das mechanisch arbeitende Fractometer bewährt.

- Leider wird dies zuwenig genutzt, vielleicht ist es zu einfach!?

- Fortsetzung wird es in „intelligenten“ Fixateurteilen finden (siehe unten).

\section{Hautstanze (Abb.2)}

Hier handelt es sich um eine empirische Untersuchung. Von Gerngroß empfohlen und bereits bei Tierversuchen von Gerngroß und Claes angewendet, war die Frage zu klären, ob es günstig ist, statt einer Inzision für die Schanzsche Schraube eine definierte Hautstanze durchzuführen.

Die empirische Anwendung zunächst im Bundeswehrkrankenhaus Ulm und in der BG-Unfallklinik Tübingen zeigte gute Erfolge. Eine weitere Dokumentation in diesen Kliniken hat dazu geführt, dass auch im St. Georg Krankenhaus in Hamburg (Eggers und Kisse) die Hautstanze zunehmend Verwendung findet.

Die empirische Erfahrung zeigt, dass die Hautstanze dann empfohlen werden kann, wenn beim Repositionsmanöver keine größeren Verschiebungen zu erwarten sind, wie dies zum Beispiel bei stark dislozierten Oberschenkelfrakturen der Fall sein kann. Sollte einmal nach einer Hautstanze eine Verschiebung eingetreten sein, lässt diese sich dann allerdings genauso wie eine Inzision in die gegebene Richtung erweitern und der versehentlich angelegte Schnitt bzw. in diesem Fall der runde Stanzdefekt durch einfache Hautnaht vernähen.

Die Gruppenmitglieder haben beschlossen, dass es sich für diese Sache nicht lohnt bzw. es auch nicht möglich sein wird, eine wissenschaftliche oder gar prospektive Studie durchzuführen. Dafür spricht zum Beispiel, dass neben diesem Parameter viel mehr andere Variablen während solch einer Untersuchung zu erwarten sind, und dass die Infektionsrate und die weitere Komplikation mit den Schanzschen Schrauben inzwischen so gering sind, dass ein signifikanter Unterschied erst bei sehr hohen Fallzahlen zu erwarten wäre.

Mit einer Hautstanze sind ideale Eintrittspforten für die Pins vom Fixateur externe möglich.

\section{MEFISTO (Monolaterales Externes Fixationssystem für Trauma und Orthopädie) (Abb. 3)}

Es handelt sich um eine Entwicklung für einen monolateralen Fixateur externe in der sog. „Body-Variante“. Diese Fixationssysteme sind auf dem Markt sehr erfolgreich, weil sie für Schaftfrakturen von einer Großzahl der Chirurgen gegenüber modulen „Baukastensystemen“ bevorzugt werden. Im Orthopädiebereich mit Angulation und Segmenttransport können die monolateralen Bodysysteme ihre größere Stabilität des Längsträgers gegenüber den modulen 11-mm-Rohren ausspielen.

Das von der AO jetzt vorgestellte System wurde in Zusammenarbeit zwischen Prof. Claes, Prof. Gerngroß und der Firma Stratec entwickelt. Nach der ersten Entwicklungsstufe wurde das System dann in den Handlings- und später den Anwendungstests durch die Arbeitsgruppe Externe Fixation genommen. Während der Phase wurden noch Modifikationen vorgenommen, bis die Testkliniken BG-Unfallklinik Tübingen und BG-Unfallklinik Ludwigshafen den klinischen Test übernommen haben.

Zunächst wurden die Traumamodule und im zweiten Anlauf die Orthopädiemodule getestet. Die Traumamodule konnten Mitte 1998, die Orthopädiemodule Anfang 1999 durch das AO LBTK und AOTK-Approval (offizielle AO/Synthes Genehmigungsverfahren) gebracht werden.

1999 und 2000 sind jetzt weitgehend alle Module fertiggestellt und im Katalog an- 


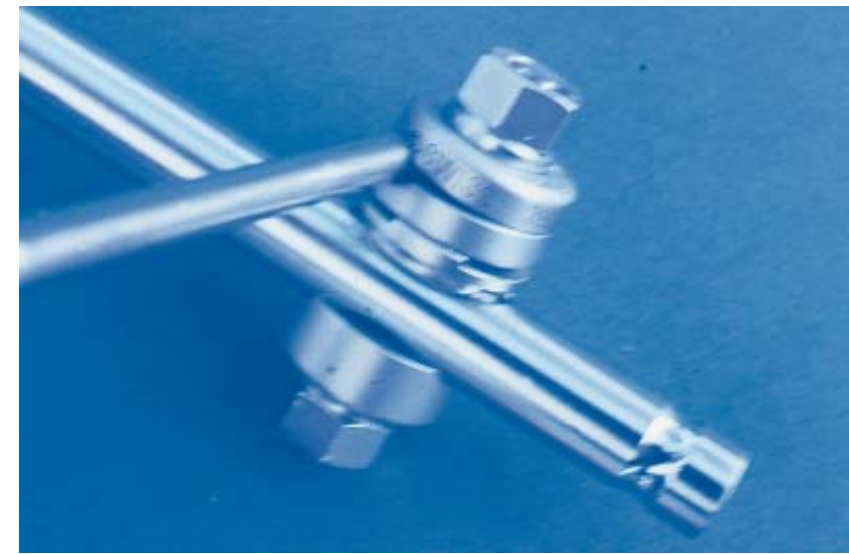

Abb. 4 Neue offene Universalbacke mit Snap-on-Effekt für das Rohr oder Stab und für Schanz'sche Schrauben 4-6mm. Das Aufsetzen ist erleichtert. Die Backen bleiben in Position, rutschen aber für die Manipulationen.

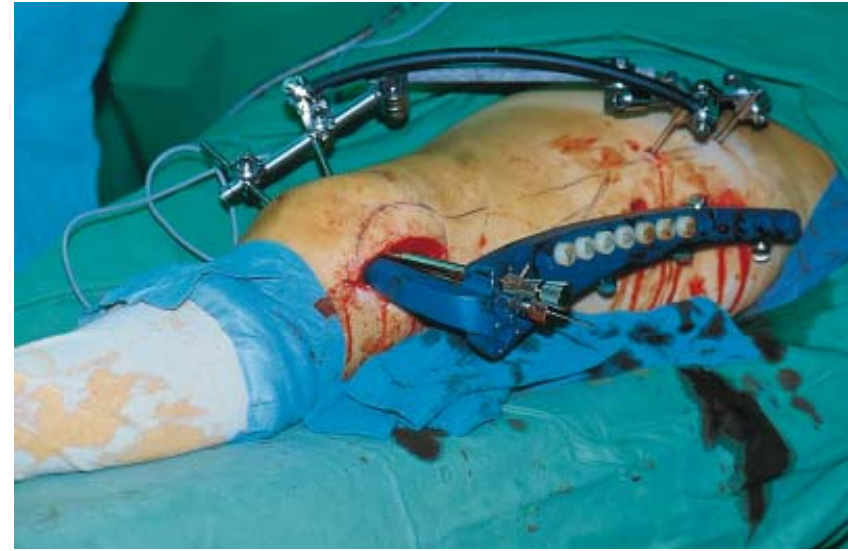

Abb.5 Die Fixateur externe 3-Rohr-Montage wird nicht als Fixateur, sondern als temporäres intraoperatives externes Repositionsinstrumentarium verwendet. Wichtige Bemerkung: Es muss die modulare 3-RohrTechnik absolut konsequent angewendet werden, dann hat man ideale Verhältnisse für Reposition und Fixation. geboten und erhältlich:

- MEFISTO Traumamodul mit $22 \mathrm{~mm}$ Kohlefaserstab

- hierzu alle Backen

- MEFISTO moduler Längsträger mit Distraktion, Kompression und Dynamisierungsmöglichkeit

- MEFISTO für Trauma und Orthopädie:

verschiedene Einzelbacken: T-Backe, Anstoß an Hybrid-Fixateur, einzelne Aufsteckbacke

- MEFISTO Orthopädiemodul für Kallotaxis

— Verlängerung und Segmenttransport mit entsprechenden Rohrteilen für den gewindetragenden Längsträger.

- So ist nach den Beschlüssen der zuständigen Export-Groups und letztendlich AO TK das Kapitel MEFISTO mit Einführung in den Katalog weitgehend abgeschlossen.

Mit dem monolateralen Mefisto Fixateur externe gibt es für Traumatologie und Orthopädie eine

$\square$ ideale Kombination eines einfachen Fixateur externe

- automatisch eingebauter Modulartechnik und

- komplexen Inhalten und Zusatzteilen für die gesamte Wiederherstellungschirurgie.

Neue offene Snap-on-Universal-Backe (Abb.4)

Als vor Jahren die offene Universalbacke entwickelt wurde, war schon einmal versucht worden, eine Lösung mit einer Feder herzustellen. Wie sich heute herausstellt, haben wir damals einen Fehler begangen, die gleich von den „Technikern“ vorgebrachten Argumente als zu großes
Hindernis anzusehen: Die Argumente damals waren: zu viele Bauteile, dadurch bedingt höhere Kosten, Notwendigkeit, die Öffnung zu groß zu machen, damit Verlust an Haltekraft, an der damals besten Backe gemessen. Gerade die letzte Frage hätten wir etwas toleranter besprechen können, weil nämlich nie eine Fixateur externe-Backe allein in einem System steht, sondern die Addition zum Rahmen. Insofern hätte man bei der Haltekraft Abstriche machen können, um die Öffnung genügend groß zu gestalten und die Haltekraft etwas zu reduzieren. So war die erste Version der offenen Universalbacke nur mit einer kleinen Tellerfeder versehen, welche die Zähne auseinanderrückt, bevor endgültig arretiert wird.

Andere Backensysteme und vor allem auch die technische Lösung der Combination Clamp (siehe Kombinationsbacke) sowie deren Markterfolg haben dazu geführt, dass nun doch rasch an eine Modifikation bzw. Weiterentwicklung der offenen Universalbacke gedacht wurde. Als erste Version wurde das jetzt vorhandene 2-Mutter-Modell in die Weiterentwicklung genommen. Es hat sich gezeigt, dass mit geringen Änderungen, zum Beispiel Verlängerung des Bolzens, einer langhubigen Feder, einer geringen Erweiterung der Öffnung, ein gutes System gefunden worden ist. Diese Version wurde bei der letzten Sitzung der Arbeitsgruppe ausgiebig einem Handlingtest unterworfen. Inzwischen wurde die Backe auch der Longbone-TK und der AOTK vorgestellt und in die Weiterbildung durch diese Gruppe genommen. Alle Ärzte, nicht nur die der Arbeitsgruppe, haben die Backe für sehr gut empfunden.
In diesem Zusammenhang wurde auch wiederholt die Frage nach einer „1-Mutter-Backe“ oder „1-Mutter-Backe“ gestellt. Besonders erfahrene Operateure und solche, welche sehr komplexe Fixateurrahmen anwenden, sind mit dem 2 Mutter-Modell durchaus zufrieden. Das 1-Mutter-Modell hat den Vorteil, dass mit einer Mutter beide Funktionen angezogen werden, wo man beim 1-MutterModell, welches in der schrittweisen Anwendung variabler ist, eben zwei Arbeitsschritte benötigt.

Diese Frage bleibt im Ergebnis unbeantwortet. Um das Projekt voranzubringen, empfiehlt die Arbeitsgruppe Externe Fixation, jetzt das 2-Mutter-Modell weiterzuentwickeln und später zu entscheiden, ob parallel oder ersatzweise ein 1-MutterModell folgen soll. Die Bemühungen haben jetzt zur Aufnahme in den europäischen Syntheskatalog geführt. Die Backe bewährt sich.

Die offene Universalbacke für das $11 \mathrm{~mm}$ Rohrsystem hat nun einen weiteren groBen Vorteil:

- Aufschnappbar („Snap-on“)

selbsthaltend

- Dadurch: Handling wesentlich einfacher.

\section{Derzeit laufende Projekte}

\section{Externe Repositionssysteme (Abb.5)}

Die 3-Rohr-Modulartechnik ist jetzt Standard bei der Schulung.

Die Diskussionen und der Erfahrungsaustausch in der Arbeitsgruppe hat gezeigt, dass eine konsequent durchgeführte 3Rohr-Modulartechnik mit geschickt ge- 
setzten Schanzschen Schrauben eine der besten, preiswertesten und am meisten verfügbarsten Repositionssysteme darstellen kann. Es wurden eingehende Gespräche mit den Produzenten Mathys Bettlach und Stratec Waldenburg geführt, dies evtl. in das Schulungs- und Setprogramm der neuen internen Fixationssysteme wie LISS einzuführen.

Des Weiteren wird noch einmal das parallel zum MEFISTO-System entwickelte Repositionsinstrument wiederbelebt und soll nach zur Verfügungstellung für Gerngroß in Ulm noch einmal getestet und evtl. verbessert werden.

- Das Fixateur externe Rohrsystem stellt bereits heute ein ideales intraoperatives temporäres Repositionssystem dar.

Allerdings nur, wenn die 3-Rohrmodulartechnik konsequent, einfach und technisch richtig angewendet wird!

\section{Bewegungsbacken}

Die Arbeitsgruppe arbeitet seit langem an Bewegungsbacken. Die Bewegungsbakken am Ellenbogen, besonders an der BG Unfallklinik Ludwigshafen, haben weitere Fortschritte gemacht. Es ist eine Veröffentlichung des Entwicklers Schmickal aus Ludwigshafen in der Zeitschrift „Der Unfallchirurg“" erschienen.

Bewegungsbacken Finger- und Handgelenk: In weiterer Entwicklung

Bewegungsbacke Sprunggelenk

Ausführliche Untersuchungen unseres Mitgliedes Schmickal aus Ludwigshafen über sehr komplexe Bewegungen im oberen Sprunggelenk, welche durch eine gefederte und variabel geführte Backe überbrückt werden soll. Erste Berechnungen sind angestellt und eine Zusammenarbeit mit einem Institut. Desgleichen sind hier Drittmittel über das Berufsgenossenschaftliche Institut für Traumatologie der Berufsgenossenschaften in die Wege geleitet. Dies verspricht ein sehr schwieriges, aber für besondere Fälle erfolgreiches Projekt zu werden.

\section{Röntgendurchlässige Kunststoffbacke für das $4 \mathrm{~mm}$ externe Fixationssystem}

Hier wird in der nächsten Zeit eine Entwicklung von unserem Mitglied Grass aus Dresden weiterverfolgt. Von diesem wurde ein erster Prototyp einer röntgendurchlässigen Backe für das 4-mm-System vorgestellt und die ersten Anwendungen gezeigt. Das System erscheint so

\section{Tabellarische Zusammenfassung}

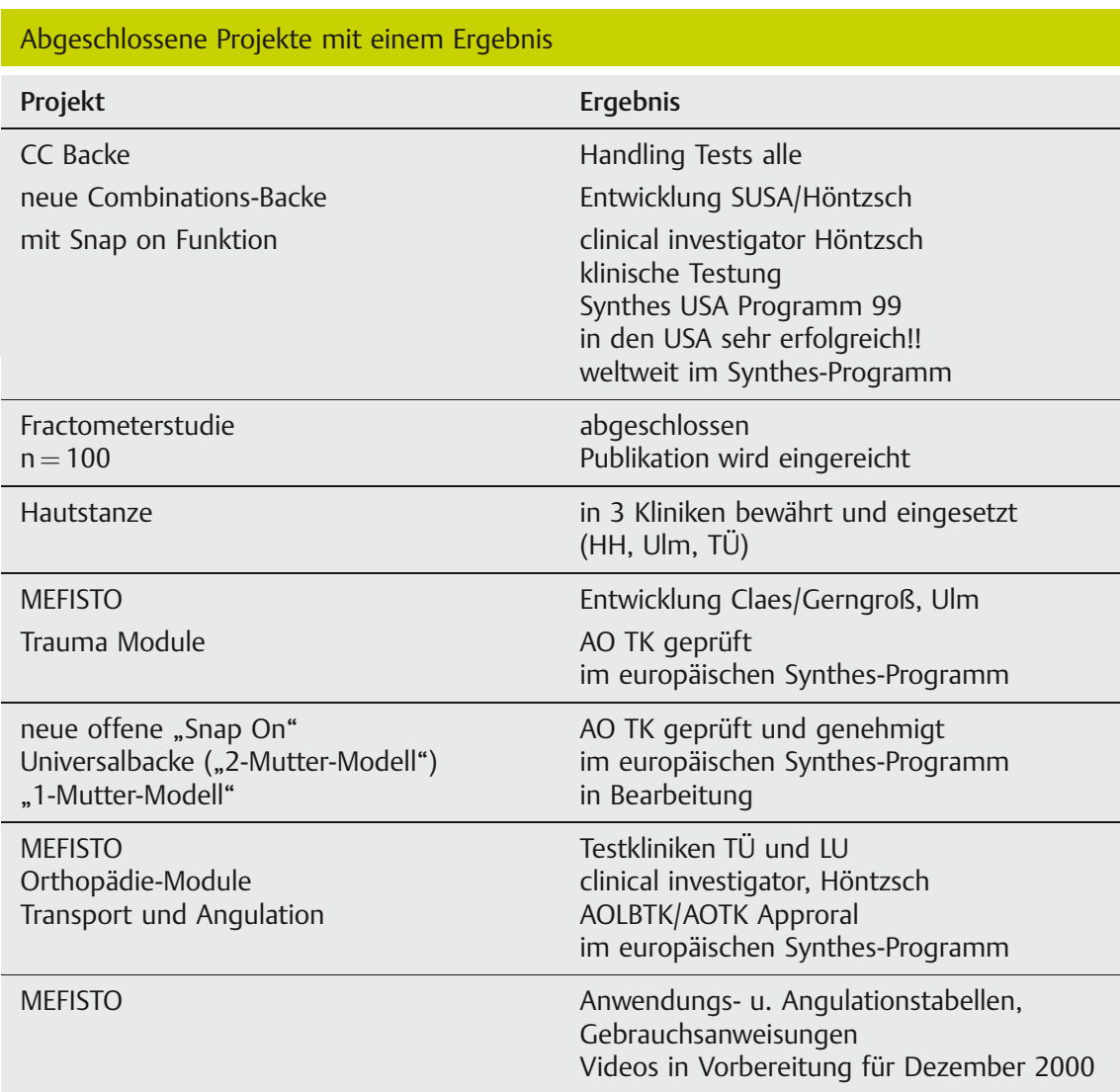

\begin{tabular}{|c|c|}
\hline Projekt & Bemerkungen \\
\hline AO EX FIX & Auftragsarbeit der $\mathrm{AO}$ \\
\hline Manual & $\begin{array}{l}\text { Publishing/Davos } \\
\text { Vorbereitungen angelaufen } \\
\text { Höntzsch, alle Mitglieder sowie weitere } \\
\text { internationale Autoren }\end{array}$ \\
\hline $\begin{array}{l}\text { externe Repositionssysteme } \\
\text { vorhandene } \\
\text { neue } \\
\text { komplexe/einfache } \\
\text { umfunktionierte }\end{array}$ & $\begin{array}{l}\text { Thema seit } 3 \text { Jahren, derzeit höchst aktuell } \\
\text { geworden. } \\
\text { Zusammenarb. und Koordinat. } \\
\text { notwendig u. in die Wege geleitet. z. B. mit } \\
\text { AO Entwicklungsinstitut in Davos }\end{array}$ \\
\hline $\begin{array}{l}\text { externe Reposition u. Fixation } \\
\text { mit rechnergestützter } \\
\text { Navigation }\end{array}$ & $\begin{array}{l}\text { Projekt Medivision - TÜ u. LU } \\
\text { Projekte Ulm, TÜ u. LU geplant; Anfragen für } \\
\text { weitere Drittmittel angedacht }\end{array}$ \\
\hline $\begin{array}{l}\text { SELDRILL } \\
\text { selbstschneidende } \\
\text { selbstbohrende } \\
\text { Schanzsche Schrauben }\end{array}$ & $\begin{array}{l}\text { klinische Evaluation, retrospekt. } \\
\text { Umfrage eingeleitet, } \\
\text { retro- und prospektive Fragebogenaktion in } \\
\text { Vorbereitung zum späteren Start. }\end{array}$ \\
\hline $\begin{array}{l}\text { Bewegungsbacken } \\
\text { Ellenbogen } \\
\text { Finger } \\
\text { Sprunggelenk }\end{array}$ & $\begin{array}{l}\text { Entwicklung Schmickal, LU } \\
\text { Prototypenbau finanziert } \\
\text { erste erfolgreiche Anwendungen } \\
\text { LU, Dresden, (TÜ) }\end{array}$ \\
\hline $\begin{array}{l}\text { röntgendurchlässige Kunststoffbacke für } 4 \\
\text { mm System }\end{array}$ & $\begin{array}{l}\text { Entwicklung Grass, Dresden } \\
\text { erster Prototyp; weitere werden finanziert, } \\
\text { breitere klinische Testung in die Wege geleitet }\end{array}$ \\
\hline
\end{tabular}




\begin{tabular}{|c|c|}
\hline Projekt & Ergebnis \\
\hline „intelligente“ Längsträger für Fix Ex & $\begin{array}{l}\text { Entwicklung Schmickal, LU } \\
\text { Unterstützung durch Arbeitsgruppe } \\
\text { sehr interessantes Projekt } \\
\text { mit Drittmitteln unterstütztes Forschungs- } \\
\text { vorhaben }\end{array}$ \\
\hline elastische Zwischenstücke & $\begin{array}{l}\text { Vorschläge Oberli, Salomonen und Fernan- } \\
\text { dez, Montevideo eher hintenangestellt }\end{array}$ \\
\hline axial verschiebbare Schanzschraube & $\begin{array}{l}\text { Vorschlag Oberli, Salomonen } \\
\text { Prototypen verschiedener Art in Bearbeitung } \\
\text { Zusammenarbeit mit AO Entwicklungsinst. } \\
\text { Davos und Mathys Bettlach }\end{array}$ \\
\hline Kühlbohrbüchse „cooling tank“ & $\begin{array}{l}\text { automatische Kühlung beim Bohren, besser } \\
\text { als ungekühlt. } \\
\text { Projekt Höntzsch, TÜ } \\
\text { Tank im Griff } \\
\text { Prototypen und Zusammenarb. mit AO Ent- } \\
\text { wicklungsinst. u. Mathys Bettlach } \\
\text { erster Einsatz erfolgreich bzw. problemlos }\end{array}$ \\
\hline Flexafix & $\begin{array}{l}\text { ein altes Projekt wird neu geprüft, } \\
\text { schwimmendes Gelenk für z. B. Handgelenks- } \\
\text { Fix. ext. }\end{array}$ \\
\hline $\begin{array}{l}\text { Koordination und „Familienbildung“ } \\
\text { aller Fix. externe-Systeme }\end{array}$ & $\begin{array}{l}\text { Vorschläge der Arbeitsgruppe liegen vor. } \\
\text { AO TK geprüft. Umsetzung und Einsicht der } \\
\text { Firmen steht aus. }\end{array}$ \\
\hline beschichtete Schanzschraube & $\begin{array}{l}\text { neuer Anlauf mit Prof. Dávid, Wuppertal } \\
\text { Wert und Nutzen beschichteter Schanz- } \\
\text { schrauben zu evaluieren, zu testen und ggf. } \\
\text { Einführen als zusätzliche Schanzschraube } \\
\text { vorbereiten. }\end{array}$ \\
\hline
\end{tabular}

erfolgreich, dass eine Weiterverfolgung angezeigt ist und von der Arbeitsgruppe durchgeführt wird. Die Tests würden auf mehrere Kliniken ausgedehnt werden.

\section{Geplante Projekte}

Externe Reposition und Fixation mit rechnergestützter Navigation

Dieses Projekt wird weiter verfolgt und im Jahr 2000 bei weiterer Verfügbarkeit, besonders der entsprechenden Module des MediVisions-Systems an der BG Unfallklinik Ludwigshafen und besonders an der BG Unfallklinik Tübingen fortgesetzt werden.

\section{Intelligentes „Zwischenstück“}

Hier sind interessante Ansätze von unserem Mitglied Schmickal mit Hilfe von erheblichen Drittelmitteln in die Wege geleitet worden, welche erlauben, über das dritte Zwischenrohr eingebaute Druck- und Zugmessstreifen den Elastizitäts- und damit den Heilungsverlauf zu verfolgen. Aus Schutzgründen für den $\mathrm{Au}-$ tor kann hier noch nicht weiter berichtet werden.
Selbstschneidende, selbstbohrende Schanzsche Schrauben

Hier wird die Arbeitsgruppe in der klinischen Evaluation und prospektiven Untersuchung mit Unterstützung der Produzenten weiter tätig sein. Es werden retround prospektive Fragebogenaktionen und Marktanalysen fortgesetzt. Erste Einsätze sind hier gelaufen. Bei der AO-Frühjahrstagung, Mai 2000, wurden die Mitglieder des 3-Länder-Treffens aus der Schweiz, Österreich und Deutschland hierzu befragt. Die Bestrebungen haben bereits dazu geführt, dass die Spitze eine Modifikation erhalten hat, welche von Mathys Bettlach als Prototyp entwickelt wurde.

\section{Bohrbüchse mit automatischer Kühlung (Cooling-tank)}

Es ist unbestritten, dass das Kühlen von Bohrungen nicht nur beim Fixateur von Vorteil ist. Hierzu ist vom Mitglied Höntzsch eine Bohrbüchse entwickelt worden, die in ihrem Handgriff einen Tank hat, welcher Kühlflüssigkeit aufnehmen kann, welcher dann beim Bohren durch leichtes Drücken oder durch „Selbstabholen“ durch den rotierenden Bohrer mit einer geringen, aber ausreichenden Menge gekühlt wird. Die Prototypen wurden vom AO Entwicklungsinstitut (Leitung M. Mehli) Davos hergestellt.

\section{Elastisches Zwischenstück}

Vorschlag: Oberli/Salomon Islands und Fernandez/Montevideo

Um in Einzelfälle mehr Elastizität in den Fixateur zu bekommen wurde vorgeschlagen, ein dünneres Z wischenrohr einzubauen. Hierfür sind Berechnungen anzustellen. Das Projekt wird allerdings nicht hoch eingeschätzt.

\section{Quere axiale Verschiebung von Schanzschen Schrauben}

Vorschlag: Oberli/Salomon Islands

In Einzelfällen ist es einmal notwendig, Schanzsche Schrauben bei liegendem Fixateur axial, d.h. in Längsrichtung der Schanzschen Schraube entweder zur Fraktur oder weg davon zu bewegen. Hierfür wurden von der Firma Mathys Bettlach 2 Prototypen zusammengestellt. Zeichnungen von Oberli, Salomon Islands stehen zur Verfügung. Dieses Projekt wird als geplantes Projekt fortgesetzt.

\section{Flexafix}

Ein altes Projekt wird neu geprüft. Schwimmendes Gelenk für z. B. Handgelenks-Fixateur externe.

\section{Koordination und „Familienbildung“ aller Fixateursysteme}

Vorschläge der Arbeitsgruppe liegen vor. Von der AO TK wurden diese geprüft. Eine Umsetzung und Einsicht der Firmen steht noch aus.

\section{Beschichtete Schanzsche Schrauben}

Neuer Anlauf mit Prof. Dávid, Wuppertal, Wert und Nutzen beschichteter Schanzscher Schrauben zu evaluieren, zu testen und ggf. Einführen als zusätzliche Schanzsche Schraube vorbereiten.

Prof. Dr. med. D. Höntzsch

Leitender Arzt

BG Unfallklinik

Schnarrenbergstr. 95

72076 Tübingen 